\title{
Interlaboratory Comparisons of NbTi Critical Current Measurements
}

\author{
A. Godeke, D. Turrioni, T. Boutboul, N. Cheggour, A. K. Ghosh, L. F. Goodrich, M. Meinesz, and A. den Ouden
}

\begin{abstract}
We report on a multi-institute comparison of critical current data measured on a modern NbTi wire for the Large Hadron Collider (LHC), which has shown a standard deviation below $1 \%$ in critical current density spread in more than 1500 measurements. Interlaboratory comparisons on $\mathrm{Nb}_{3} \mathrm{Sn}$ wires have shown ambiguities that could be attributable to strain related differences in critical current density, originating from differences in sample handling, reaction, and mounting techniques, or also to differences in the magnetic field and current calibrations between the institutes. A round robin test of a well characterized NbTi wire provides a baseline variance in critical current results that is presumed to be attributable only to differences in the characterization systems. Systematic differences on the order of $3.5 \%$ are found in the comparison. The most likely cause for the observed differences is a small diameter holder that brings the wire into a strain regime in which strain effects can no longer be ignored. A NbTi round robin test, when performed properly, will separate system differences from sample specific differences and provide laboratories with an opportunity to calibrate equipment against a standard measurement.
\end{abstract}

Index Terms-critical current, $\mathrm{NbTi}$, round robin, standardization

\section{INTRODUCTION}

$\mathbf{M}$ ULTI-institute collaborations for the development of large magnet systems are common in the applied superconductivity community. Some examples are the International Thermonuclear Experimental Reactor (ITER), the LHC, and the U.S. LHC Accelerator Research Program (LARP). A common factor for these collaborations is that the magnet development relies on reliable and comparable measurements of the critical current $\left(I_{\mathrm{c}}\right)$ in different laboratories. Comparable $I_{\mathrm{c}}$ measurements between different laboratories are far from trivial as was clearly demonstrated during the ITER $\mathrm{Nb}_{3} \mathrm{Sn}$ wire benchmark test series in the middle of the 1990's, in

Manuscript received August 19, 2008. This work was supported by the Director, Office of Science, High Energy Physics, U.S. Department of Energy under contract No. DE-AC02-05CH11231.

A. Godeke (corresponding author; phone: +1-510-486-4356; e-mail: agodeke@lbl.gov) is with Lawrence Berkeley National Laboratory, Berkeley CA 94720, USA

D. Turrioni is with Fermi National Accelerator Laboratory, Batavia, IL 60510, USA

T. Boutboul is with CERN, AT/MCS, CH-1211, Geneve 23, Switzerland

N. Cheggour and L. F. Goodrich are with National Institute of Standards and Technology, Boulder, CO 80305 USA

A. K. Ghosh is with Brookhaven National Laboratory, Upton, NY11973, USA

M. Meinesz is with Oxford Instruments, Superconducting Technology, Carteret, NJ 07008 USA

A. den Ouden is with the Low Temperature Division, Faculty of Science and Technology, University of Twente, P.O. Box 217, 7500 AE, Enschede, The Netherlands

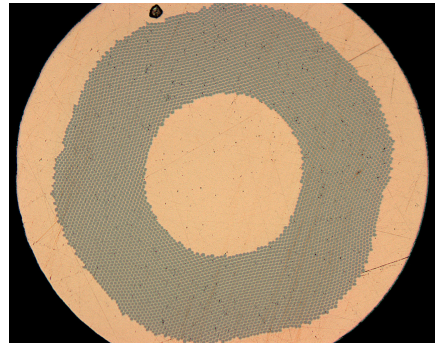

Fig. 1. Cross-section of the NbTi wire used for the $I_{\mathrm{C}}$ comparisons.

which an initial variation above 30\% [1] in multi-institute measurements of $I_{\mathrm{c}}$ led to a stringent standardization of $I_{\mathrm{c}}$ measurement procedures. The Ti-6Al-4V 'ITER barrel' that was used for the second and third benchmark rounds led to a reduction of the interlaboratory variation in $I_{\mathrm{c}}$ to less than 4\% [2], [3]. The ITER barrel has since then become the standard measurement holder for the characterization of medium current density $\left(J_{\mathrm{c}}\right) \mathrm{Nb}_{3} \mathrm{Sn}$ wires.

The $J_{\mathrm{c}}$ for modern high current $\mathrm{Nb}_{3} \mathrm{Sn}$ wires has doubled since the third ITER benchmark, and a modern $0.8 \mathrm{~mm}$ wire can carry currents approaching $500 \mathrm{~A}$ and $900 \mathrm{~A}$ at $4.2 \mathrm{~K}$ and 15 and $12 \mathrm{~T}$, respectively [4]. Such large sample currents have highlighted some potential issues with the standard ITER barrel approach. Some laboratories have, therefore, modified their setups to address these issues. These changes, the use of stainless-steel sample holders, and different sample mounting procedures, have re-introduced uncertainties in the comparison of $I_{\mathrm{c}}$ values measured in different institutes, which became evident during the U.S. LARP collaboration.

The strain sensitivity of $\mathrm{Nb}_{3} \mathrm{Sn}$ disables a separation of differences that can originate from accuracies in magnetic field, temperature and current (the experiment), and differences in sample mounting techniques. To separate experimental and sample mounting differences, and to provide a baseline comparison for the experiments in terms of magnetic field, temperature and current accuracy, we decided to do a multi-laboratory round robin comparison using an extensively characterized, exceptionally homogeneous $\mathrm{NbTi}$ wire. $I_{\mathrm{c}}(H)$ of this wires has been measured at Lawrence Berkeley National Laboratory (LBNL), Fermi National Accelerator Laboratory (FNAL), CERN, the National Institute of Standards and Technology (NIST), Brookhaven National Laboratory (BNL), the Applied Superconductivity Center, Florida State University (ASC), Oxford Instruments, Superconducting Technology (OST), and the University of Twente (UT). 
TABLE I

CERN STATISTICS FOR NBTI WIRE SPOOL 02R00056A01UX AT $T=4.222 \mathrm{~K}$, MEASURED ON 8 DIFFERENT G11 86 MM BOBBINS WITH $60 \mathrm{~N}$ MOUNTING TENSION, IN 4 NMR CALIBRATED 12 T MAGNETS (THREE OXFORD INSTRUMENTS (OI) AND ONE CRYOGENICS). FROM [5].

\begin{tabular}{|c|c|c|c|c|c|c|}
\hline$\mu_{0} H[\mathrm{~T}]$ & 2.5 & 4.0 & 5.0 & 6.0 & 7.0 & 8.0 \\
\hline Average: $I_{\mathrm{C}}[\mathrm{A}]^{*} / n$ & $871.1 / 50$ & $657.6 / 55$ & $538.8 / 52$ & $426.2 / 48$ & $313.6 / 43$ & $201.7 / 35$ \\
\hline Standard deviation: $I_{\mathrm{C}}[\mathrm{A}] / n$ & $0.0 / 0$ & $2.9 / 4$ & $2.5 / 3$ & $2.1 / 3$ & $1.9 / 3$ & $1.7 / 2$ \\
\hline Standard deviation: $I_{\mathrm{C}}[\%]$ & 0.0 & 0.4 & 0.5 & 0.5 & 0.6 & 0.9 \\
\hline Maximum: $I_{\mathrm{C}}[\mathrm{A}]^{*}$ & 871.1 & 664.3 & 545.3 & 432.2 & 318.9 & 209.4 \\
\hline Minimum: $I_{\mathrm{C}}[\mathrm{A}]^{*}$ & 871.1 & 648.8 & 531.9 & 418.4 & 305.9 & 196.5 \\
\hline$(\operatorname{Max}-\operatorname{Min}) / \operatorname{Avg}: I_{\mathrm{c}}[\%]$ & 0.0 & 2.4 & 2.5 & 3.2 & 4.1 & 6.4 \\
\hline Cooldowns / Runs & $1 / 1$ & $236 / 236$ & $238 / 239$ & $1647 / 1649$ & $1584 / 1584$ & $1582 / 1582$ \\
\hline
\end{tabular}

${ }^{*}$ At a resistive criterion of $10^{-14} \Omega \cdot \mathrm{m}$.

\section{SAMPLES}

Very large quantities of $\mathrm{NbTi}$ wire have been manufactured for the construction of the LHC at CERN. These wires have been very well characterized through stringent acceptance tests at CERN. A NbTi wire with exceptional longitudinal homogeneity (spool 02R00056A01UX, sample cuts \#265 and \#267), which was used at CERN for the qualification of the $I_{\mathrm{c}}$ tests, was selected for the present round robin test. A crosssection of the wire is shown in Fig. 1. A summary of the CERN $I_{\mathrm{c}}$ measurements on this wire, using a $86 \mathrm{~mm}$ G11 bobbin, is given in Table I. The wires, manufactured by Furukawa, have a diameter of $0.825 \mathrm{~mm}$, a $\mathrm{Cu} / \mathrm{SC}$ ratio of 1.95 , a filament diameter of $6 \mu \mathrm{m}$, and a twist pitch of $15 \mathrm{~mm}$. Over more than $1500 I_{\mathrm{c}}$ measurements on more than 100 different samples, the wire exhibits a standard deviation in $I_{\mathrm{c}}$ below $3 \mathrm{~A}$ and a standard deviation in the $n$-value of 4 or less, as indicated in Table I. The variation in $I_{\mathrm{c}}$ over $30 \mathrm{~m}$ and the long-term measurement reproducibility of typical $I_{\mathrm{c}}$ measurements on comparable wires, investigated previously at $\mathrm{BNL}$, are both below $0.5 \%$ [6].

\section{EXPERIMENT}

The $\mathrm{NbTi}$ wires were distributed amongst the participants, and each laboratory mounted the wire on its own sample holder. The central sections of all sample holders used in the present comparison are identical and based on a Ti-6Al-4V ITER barrel, with a diameter of $32 \mathrm{~mm}$, a pitch of $3.2 \mathrm{~mm}$, and an angle between the current and the applied field of $88.2^{\circ}$. The exception is NIST, which measured the NbTi wire on a $25 \mathrm{~mm}$ diameter $\mathrm{CuBe}$ Walters spring, with a pitch of $6.35 \mathrm{~mm}$ and an angle between the current and the applied field of $85.4^{\circ}$. Tests of in-field voltage versus current were performed using the common procedures in each laboratory. The helium bath temperature was recorded in all but one case to allow correction for the temperature dependence of the $I_{\mathrm{c}}$. Additional experiments were performed at BNL to investigate the difference between measuring DC or with a continuous ramping current, and to investigate the effect of different barrel materials on barrels of identical diameters.

\section{DATA REDUCTION}

The electric field as a function of current of superconductors can be described through the Anderson-Kim flux creep model [7], [8], which dictates linearity on a double logarithmic scale. This leads, in the case of an electric field criterion $\left(E_{\mathrm{c}}\right)$ for the critical current density $J_{\mathrm{c}}$, to [9]:

$$
E=E_{c}\left(\frac{J}{J_{c}(H, T)}\right)^{n(H, T)} .
$$

The value of $n$ represents the steepness of the transition and the slope on a double logarithmic scale. Some of the laboratories in the present work determined the $I_{\mathrm{c}}$ using an $E_{\mathrm{c}}$ criterion. For this case, the measured voltage-current $(V(I))$ trace is effectively fitted to:

$$
V(I)=V_{0}+R I+E_{\mathrm{c}} L\left(\frac{I}{I_{\mathrm{c}}}\right)^{n},
$$

in which $V_{0}$ is an offset voltage, $R$ is a resistance, used to fit a sometimes occurring resistive slope in a $V(I)$ transition, and $L$ is the length between the voltage tap pair. Some laboratories, and the existing CERN reference statistics, applied a resistivity criterion $\left(\rho_{\mathrm{c}}\right)$, in which case the measured $V(I)$ trace is fitted to

$$
V(I)=V_{0}+R I+I\left(\frac{\rho_{\mathrm{c}} L}{A}\right)\left(\frac{I}{I_{\mathrm{c}, \rho}}\right)^{n},
$$

where $A$ represents the cross-sectional area of the wire. Note that this criterion is more ambiguous, since it involves a choice for $A$. The criteria used for the round robin comparison were $E_{\mathrm{c}}=10^{-5} \mathrm{~V} \cdot \mathrm{m}^{-1}$ and $\rho_{\mathrm{c}}=10^{-14} \Omega \cdot \mathrm{m}$. The critical current results, derived using an electric field criterion or a resistive criterion, are related, provided that $R$ is sufficiently low to not interfere with the criterion, by a combination of (1) with Ohm's law, yielding

$$
I_{\mathrm{c}, \rho}=\left(\frac{\rho_{\mathrm{c}} I_{\mathrm{c}, E}^{n}}{E_{\mathrm{c}} A}\right)^{\frac{1}{n-1}} .
$$

In (3), $R$ was sufficiently low in all measurements, and (4) was used to translate all results to a resistive criterion.

The CERN reference data are given at a temperature $T=4.222 \mathrm{~K}$, and the results, measured at different bath temperatures $\left(T_{\text {bath }}\right)$, are corrected for this small discrepancy in temperature. Ideally, the samples should be parameterized by the use of well established scaling relations [10], but the required scaling parameters cannot be accurately determined, due to the limited data range. Also, since the temperature error is small, a limited range - linear - correction will be sufficient [6], [11]:

$$
I_{\mathrm{c}}(4.222 \mathrm{~K})=I_{\mathrm{c}}\left(T_{\text {bath }}\right)\left(\frac{T_{\mathrm{c}}(H)-4.222}{T_{\mathrm{c}}(H)-T_{\text {bath }}}\right),
$$


TABLE II

BNL COMPARISON BETWEEN BARREL MATERIALS, WIRE BATCH, AND RAMP OR DC AT $\rho=10^{-14} \Omega \cdot \mathrm{M}, T=4.222 \mathrm{~K}$, AND $20 \mathrm{~N}$ TENSION.

\begin{tabular}{|c|c|c|c|c|c|}
\hline Material & & Ti-6Al-4V & & G-10 & SS-316 \\
\hline Wire & $\# 265$ & \#265 & \#267 & \#267 & \#267 \\
\hline Ramp/DC & $\mathrm{DC}$ & $10 \mathrm{~A} \cdot \mathrm{s}^{-1}$ & DC & $\mathrm{DC}$ & $\mathrm{DC}$ \\
\hline$\mu_{0} H[\mathrm{~T}]$ & \multicolumn{5}{|c|}{$I_{\mathrm{C}}[\mathrm{A}] / n$} \\
\hline 5 & $536 / 36$ & $536 / 37$ & $538 / 36$ & $545 / 40$ & $542 / 37$ \\
\hline 6 & $425 / 35$ & $425 / 35$ & $425 / 33$ & 432 / 36 & $428 / 35$ \\
\hline 7 & $315 / 31$ & $317 / 34$ & $315 / 29$ & $324 / 33$ & $317 / 30$ \\
\hline 8 & $206 / 26$ & $207 / 29$ & $208 / 26$ & $214 / 27$ & $208 / 26$ \\
\hline 9 & & & $107 / 21$ & $111 / 19$ & $104 / 18$ \\
\hline
\end{tabular}

where:

$$
T_{\mathrm{c}}(H)=9.2\left(1-\frac{\mu_{0} H}{14.5}\right)^{0.59} .
$$

Note that relation 6 implicitly assumes $T_{\mathrm{c}}(0)=9.2 \mathrm{~K}$, $\mu_{0} H_{\mathrm{c} 2}(0)=14.5 \mathrm{~T}$ and $H_{\mathrm{c} 2}(T) / H_{\mathrm{c} 2}(0)=1-\left(T / T_{\mathrm{c}}(0)\right)^{1.7}$. Corrections for self-field were considered, but they are irrelevant for comparisons of results from holders with identical diameter and pitch. No self-field corrections were therefore applied to the results.

\section{RESUlts}

The comparison between a DC and a ramping current measurement, and the influence of various barrel materials, as measured at BNL, are summarized in Table II. We found that it makes no difference whether the $V(I)$ transition is measured in DC mode or with a ramping current. Negligible differences are observed between sections \#265 and \#267 of the spool, which is consistent with a variance below $0.5 \%$ in earlier tests [6]. Differences are observed, however, between the use of different barrel materials (all at $32 \mathrm{~mm}$ ), which specifically becomes evident when the Ti-6Al-4V data is compared to the G10 data. At 5 and $6 \mathrm{~T}$, the G10 results are $1.3 \%$ and $1.6 \%$ higher, respectively. At 7 and $8 \mathrm{~T}$, the G10 results are $2.8 \%$ higher, and at $9 \mathrm{~T}$, the G10 result is 3.7\% higher. The SS-316 results are increased by about $0.7 \%$ at 5,6 and $7 \mathrm{~T}$, similar at $8 \mathrm{~T}$, and reduced by $2.8 \%$ at $9 \mathrm{~T}$.

The overall results of the interlaboratory comparisons are given in tabulated form in Table III, and graphically for the overlapping magnetic field values in Fig. 2. For clarity, the FNAL14 and BNL8 results are omitted from the graph. When the small variations in the slope of $I_{\mathrm{c}}(H)$ are ignored, we found that the differences between the results from the participants are not random, but systematic and on the order of $3.5 \%$. Moreover, the LBNL, BNL and the CERN reference results agree, as well as the results from FNAL, new CERN (mounted with $20 \mathrm{~N}$ on a $32 \mathrm{~mm}$ Ti-6Al-4V holder as opposed to $60 \mathrm{~N}$ on a $86 \mathrm{~mm} \mathrm{G11}$ holder), OST, and UT. The NIST data are an average between those agreements. The variation in the $n$-values appears random and low, and the tendencies are not in agreement with the systematic $I_{\mathrm{C}}$ differences, with BNL providing the lowest values and LBNL the highest values, the latter representing the only results within the original CERN standard deviation.

\section{DISCUSSION}

The fact that the observed deviations are not random suggests a systematic error source in the measurements. Errors in the magnet field constants and resistors used for the current measurement will result in a slope change in the $I_{\mathrm{c}}(H)$ graph, rendering such errors unlikely. The observed parallel shift can be explained by errors in the bath temperature measurements on the order of $60 \mathrm{mK}$, which is, considering the stated uncertainties in the temperature measurement below $5 \mathrm{mK}$, unlikely. Differences in the position of the wire in the magnet with respect to the field calibration position can cause systematic differences, as are observed, but the order of deviation of 0.1 to $0.2 \mathrm{~T}$ renders this also an unlikely cause.

A likely cause for the observed systematic differences is a change in $H_{\mathrm{c} 2}$, as a result of strain. The effect of strain on the superconducting properties of NbTi is available in the literature and known to be small for axial strain values below about $1 \%$ [12]. The strain on the outside of a wire $\epsilon_{\mathrm{o}}$ with diameter $d_{\mathrm{w}}$, wound on a barrel with radius $r_{\mathrm{b}}$, assuming elastic behavior, is

$$
\epsilon_{\mathrm{o}}=\frac{r_{\mathrm{b}}+d_{\mathrm{w}}}{r_{\mathrm{b}}+0.5 d_{\mathrm{w}}}-1
$$

which leads to $1 \%, 2.5 \%$ and $3.2 \%$ tensile strain on the outside of a wire for a 86, 32, and $25 \mathrm{~mm}$ diameter barrel, respectively. In an elastic model, the neutral line does not shift, and the same magnitude of axial strain is produced compressive on the inside wire surface. In reality, the $\mathrm{Cu}$ matrix will yield, the neutral line will move towards a smaller radius, the tensile strain on the outer surface will become larger, and the compressive strain on the inside surface will become smaller. The net result of this is that tensile strains of $1 \%$ and higher can be expected in the outer NbTi filaments, bringing the wires into the tensile regime, where the strain dependence can no longer be ignored. The combined effect of tensile load during winding and a small winding radius can therefore play an important, underestimated role in the interlaboratory comparisons.

The NIST measurement was performed on a CuBe Walters spring with diameter of $25 \mathrm{~mm}$, and the significant strain difference compared to that of the $32 \mathrm{~mm}$ ITER barrel is, for now, speculative, but could bring the NIST results in better agreement with the original CERN data. A tensile strain experiment was performed by NIST on the NbTi wire on this $25 \mathrm{~mm}$ diameter spring, and the $I_{\mathrm{c}}$ at $7 \mathrm{~T}$ was observed to be reduced by about $5 \%$ at $0.5 \%$ applied tensile strain. The magnitude of change is possibly sufficient to attribute the observed $3.5 \%$ systematic change between the laboratories to small differences in the strain state of the wire. Further investigations are needed, however, focussing specifically on the strain dependence of a straight section of wire, and including a next round robin with more emphasis on comparable mounting procedures and possibly a larger diameter barrel.

It is, for now, unclear why all but one laboratories report an $n$-value that is substantially below the original CERN statistics and outside the standard deviation for these statistics. 
TABLE III

$I_{\mathrm{C}}$ AND $n$-VALUE RESULTS AT A RESISTIVE CRITERION OF $10^{-14} \Omega \cdot \mathrm{M}$.

\begin{tabular}{|c|c|c|c|c|c|c|c|c|c|}
\hline Institute & LBNL & FNAL15 & FNAL14 & CERN & NIST & BNL8 & BNL12 & OST & UT \\
\hline Magnet & $\begin{array}{l}\text { 15T Cryo- } \\
\text { magnetics }\end{array}$ & OI-15/17T & OI-14/16T & $\begin{array}{c}12 \mathrm{~T} \\
\text { Cryogenics }\end{array}$ & OI-16/18T & $\begin{array}{l}\text { American } \\
\text { Magn-8T }\end{array}$ & $\begin{array}{l}\text { 12T Cryo- } \\
\text { magnetics }\end{array}$ & OI-16T & $\begin{array}{c}15 \mathrm{~T} \\
\text { Cryogenics }\end{array}$ \\
\hline Calibration & Hall probe & Hall probe & Hall probe & NMR & NMR & NMR & - & - & - \\
\hline $\begin{array}{l}\text { Tmeas. }[\mathrm{K}]^{\mathrm{a}} \\
\text { Criterion }\end{array}$ & $\begin{array}{c}4.233 \\
10^{-5} \mathrm{~V} \cdot \mathrm{m}^{-1}\end{array}$ & $\begin{array}{c}4.216 \\
10^{-14} \Omega \cdot \mathrm{m}\end{array}$ & $\begin{array}{c}4.216 \\
10^{-14} \Omega \cdot \mathrm{m}\end{array}$ & $\begin{array}{c}4.343 \\
10^{-14} \Omega \cdot \mathrm{m}\end{array}$ & $\begin{array}{c}4.225 \\
10^{-5} \mathrm{~V} \cdot \mathrm{m}^{-1}\end{array}$ & $\begin{array}{c}4.215 \\
10^{-14} \Omega \cdot \mathrm{m}\end{array}$ & $\begin{array}{c}4.228 \\
10^{-14} \Omega \cdot \mathrm{m}\end{array}$ & $\begin{array}{c}\text { not rec. } \\
10^{-5} \mathrm{~V} \cdot \mathrm{m}^{-1}\end{array}$ & $\begin{array}{c}4.238 \\
10^{-5} \mathrm{~V} \cdot \mathrm{m}^{-1}\end{array}$ \\
\hline DC / Ramp & DC & DC & DC & $12.5 \mathrm{~A} \cdot \mathrm{s}^{-1}$ & DC & $5 \mathrm{~A} \cdot \mathrm{s}^{-1}$ & DC & DC & DC \\
\hline$\mu_{0} H[\mathrm{~T}]$ & \multicolumn{9}{|c|}{$I_{\mathrm{C}}[\mathrm{A}] / n$ at $\rho=10^{-14} \Omega \cdot \mathrm{m}$ and $T=4.222 \mathrm{~K}$} \\
\hline 1 & & $1385 / 33$ & & & & & & & \\
\hline 2 & & $991 / 37$ & 996 / 47 & & & & & & \\
\hline 3 CERN Ref: & & $787 / 40$ & $785 / 38$ & & & & & & \\
\hline $4 \Rightarrow 658 / 55$ & & $647 / 43$ & $644 / 40$ & & $650 / 46$ & & & & \\
\hline 9 & & $92 / 20$ & $89 / 16$ & & $96 / 20$ & & & $92.3 /-$ & $88 / 21$ \\
\hline 10 & & & & & $21 / 10$ & & & & \\
\hline
\end{tabular}

${ }^{a}$ Recorded temperature accuracies below $5 \mathrm{mK}$.

${ }^{\mathrm{b}}$ During sample mounting. A range indicates estimated values.
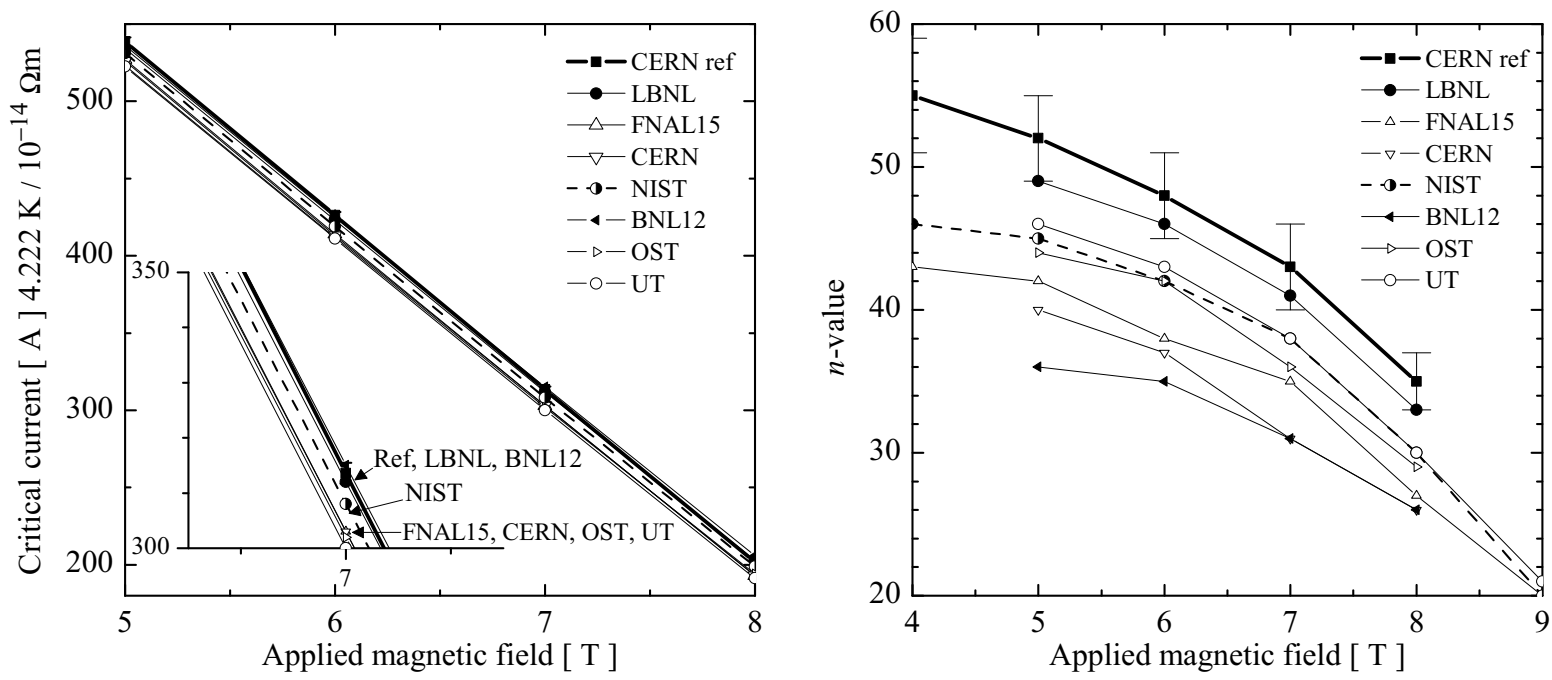

Fig. 2. $\quad I_{\mathrm{C}}$ (left) and $n$ (right) as functions of applied magnetic field. The inset in the left graph is an enlargement around $7 \mathrm{~T}$.

\section{CONCLUSION}

A round robin test of critical current measurements was performed on a homogeneous NbTi wire in 7 participating laboratories. The comparison highlights systematic differences in the critical current between laboratories, which are on the order of $3.5 \%$. These systematic differences are attributable most likely to the use of a measurement holder of small diameter and a resulting strain dependence of the $\mathrm{NbTi}$, rendering the experiment sensitive to the wire tension during mounting. Further research is required to investigate the axial strain onset of non-negligible strain dependence in NbTi wires. This will determine the best method for a more consistent mounting procedure for a second round robin, in which the systematic errors should become negligible.

\section{REFERENCES}

[1] P. Bruzzone, "Bench Mark Test on $\mathrm{Nb}_{3} \mathrm{Sn}$ strands," ITER JCT, Final summary report April 93 - May 94, 1994.

[2] H. G. Knoopers et al., "Third round of ITER $\mathrm{Nb}_{3} \mathrm{Sn}$ strand bench mark test," Inst. of Physics Conference Series, vol. 158, p. 1271, 1997.

[3] P. Bruzzone, "The 2. round of strand Bench Mark Test," ITER JCT, Final Report July 94 - August 95, 1995.

[4] A. Godeke et al., "Short sample estimate for HD2a," Lawrence Berkeley National Laboratory, Tech. Rep. Draft, 2007.

[5] T. Boutboul, Privat communication.

[6] A. Ghosh and E. Sperry, "NbTi CERN-reference wire measurements at BNL," Brookhaven National Laboratory, Tech. Rep. 649-39 (AM-MD349), 2007.

[7] P. W. Anderson, Phys. Rev. Lett., vol. 9, p. 309, 1962.

[8] Y. B. Kim, C. F. Hempstead, and A. R. Strnad, Phys. Rev. Lett., vol. 9, p. $306,1962$.

[9] A. Godeke, "Performance boundaries in $\mathrm{Nb}_{3} \mathrm{Sn}$ superconductors," Ph.D. 
dissertation, Univ. of Twente, Enschede, The Netherlands, 2005, ISBN 90-365-2224-2.

[10] L. Bottura, IEEE Trans. Appl. Supercond., vol. 10, no. 1, p. 1054, 2000.

[11] M. S. Lubell, "Empirical scaling formulas for critical current and critical fields of commercial NbTi," IEEE Trans. Magn., vol. MAG-19, no. 3, p. 754, 1983.

[12] J. W. Ekin, "Relationships between critical current and stress in NbTi," IEEE Trans. Magn., vol. MAG-23, no. 2, p. 1634, 1987. 